\title{
The Neurogenesis-Controlling Factor, Pax6, Inhibits Proliferation and Promotes Maturation in Murine Astrocytes
}

\author{
Katsuyasu Sakurai ${ }^{1}$ and Noriko Osumi ${ }^{1,2}$ \\ ${ }^{1}$ Division of Developmental Neuroscience, Center for Translational and Advanced Animal Research, Tohoku University Graduate School of Medicine, \\ Sendai 980-8575, Japan, and ${ }^{2}$ Core Research for Evolutional Science and Technology, Japan Science and Technology Corporation, Kawaguchi 332-0012, \\ Japan
}

\begin{abstract}
Astrocytes serve various important functions in the CNS, but the molecular mechanisms of their generation and maturation are still enigmatic. Here, we show that Pax6, a key transcription factor that controls neurogenesis, also regulates proliferation, differentiation, and migration of astrocytes in the CNS. We first reveal that Pax6 is expressed in astrocytes during development as well as postnatally in the wild-type mouse. Astrocytes derived from Pax6 homozygous mutants (Sey/Sey) mice exhibited aberrant proliferation together with immature differentiation, both in vivo and in vitro, with higher migration potential in scratch-wound assays in vitro. Furthermore, a larger population of Sey/Sey astrocytes expresses neural stem cell markers such as nestin, Sox2, and prominin-1. These phenotypes of Pax6-deficient astrocytes putatively occur via higher Akt activity. Thus, the breakdown of Pax6 function induces the retention of neural stem-like characteristics and inhibits astrocyte maturation.
\end{abstract}

Key words: Pax6; astrocyte; differentiation; gliogenesis; maturation; proliferation

\section{Introduction}

Astrocytes are brain glial cells that provide structural and trophic support for neurons, formation of the blood-brain barrier, and regulation of synaptogenesis and synaptic transmission, thereby contributing to neurological and psychiatric diseases (Cotter et al., 2001; Allen and Barres, 2005; Volterra and Meldolesi, 2005). In the developing cortex, both neurons and astrocytes are generated from neural stem cells, although production of the former cells precedes the latter ones. It has recently been noticed that the astrocytes share similar characteristics with the neural stem cells. For example, adult neural stem cells in the subventricular zone (SVZ) along the walls of the lateral ventricles and the subgranular zone (SGZ) within the dentate gyrus of the hippocampus have astrocyte-like characteristics, including expression of glial fibrillary acid protein (GFAP) (for review, see Ihrie and AlvarezBuylla, 2008). Moreover, 70\% of genes expressed in Muller glial cells in the retina (equivalent to astrocytes in the CNS) are similar to those expressed in retinal progenitor cells, whereas most other types of retinal cells have only $\sim 10 \%$ overlap with retinal progenitors (Blackshaw et al., 2004).

\footnotetext{
Received Nov. 15, 2007; revised March 10, 2008; accepted March 14, 2008.

This work was supported by the Core Research for Evolutional Science and Technology from the Japanese Science and Technology Agency (N.O.). K.S. is a Japan Society for the Promotion of Science research fellow (DC1). We thank Drs. Magdalena Gotz, Veronica van Heyningen, Keiko Nakayama, and Noriko Ishida for their helpful advice and thoughtful discussions, and Drs. Wieland Huttner, Masahiko Watanabe, and Yuji Owada, and Mineko Kengaku for providing valuable antibodies and a plasmid. We also thank Ayumi Ogasawara for the maintenance of the Sey/+ mouse colony and technical assistance.

Correspondence should be addressed to Noriko Osumi, Division of Developmental Neuroscience, Center for Translational and Advanced Animal Research, Tohoku University Graduate School of Medicine, 2-1, Seiryo-machi, Aobaku, Sendai 980-8575, Japan. E-mail: osumi@mail.tains.tohoku.ac.jp.

DOI:10.1523/JNEUROSCI.5074-07.2008

Copyright $\odot 2008$ Society for Neuroscience $\quad$ 0270-6474/08/284604-09\$15.00/0
}

The Pax6 gene encodes a transcription factor that contains a paired-domain and a homeodomain, and is involved in peripheral and CNS development (for review, see Prosser and van Heyningen, 1998; Osumi, 2001; Simpson and Price, 2002). In the developing rodent CNS, Pax6 is strongly expressed in neural stem/progenitor cells, and plays pivotal roles in brain patterning, neuronal specification, neuronal migration, and axonal projections (Osumi, 2001; Simpson and Price, 2002). Postnatally, Pax6 is not expressed in cortical neurons, but is expressed in certain types of neurons in the cerebellum, amygdala, and olfactory bulb (Kawano et al., 1999; Yamasaki et al., 2001; Hack et al., 2005; Kohwi et al., 2005; Tole et al., 2005). Our group has recently reported that Pax6 is expressed in GFAP-positive $\left(\mathrm{GFAP}^{+}\right)$neural stem/early progenitor cells in the rat hippocampal dentate gyrus, and that Pax6 regulates cell proliferation and differentiation in these cells (Maekawa et al., 2005). In the course of this study, we also found that Pax6 is expressed in GFAP ${ }^{+}$astrocytes in the rat hippocampus. We also noticed that Pax6 expression overlaps with glutamate-aspartate transporter (GLAST), a marker for astrocytic precursors in the mouse spinal cord (Ogawa et al., 2005). Furthermore, the expression patterns of Pax6 in the Allan Brain Atlas imply that astrocytes may express Pax6 in the adult mouse CNS (http://brain-map.org/welcome.do) (Lein et al., 2007). Such evidence has prompted us to examine how Pax6 is involved in development of astrocyte progenitors and/or mature astrocytes.

Here we identified, in the wild-type mouse, expression of Pax6 by astrocyte progenitors and matured astrocytes of the CNS in vivo and in vitro. To address the role of Pax6 in astrocytes, we performed the primary culture of astrocytes taken from wild-type and Pax6-deficient, homozygous mutant Sey/Sey mice (Hill et al., 1991), and investigated the cell proliferation, maturation, and 
A

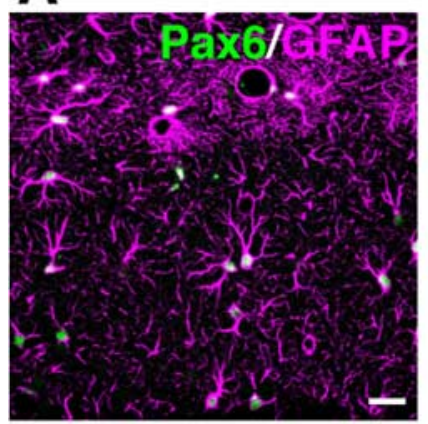

B

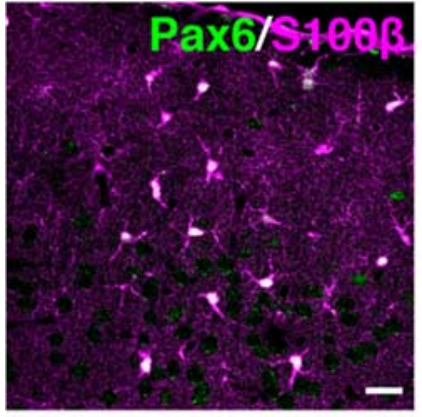

C

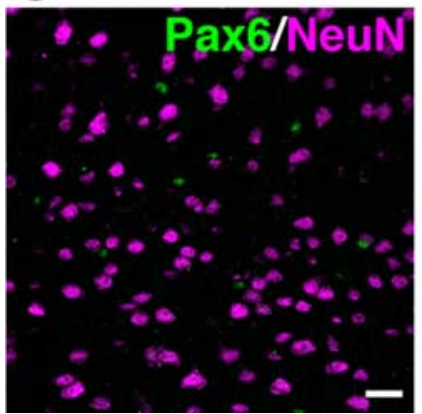

A
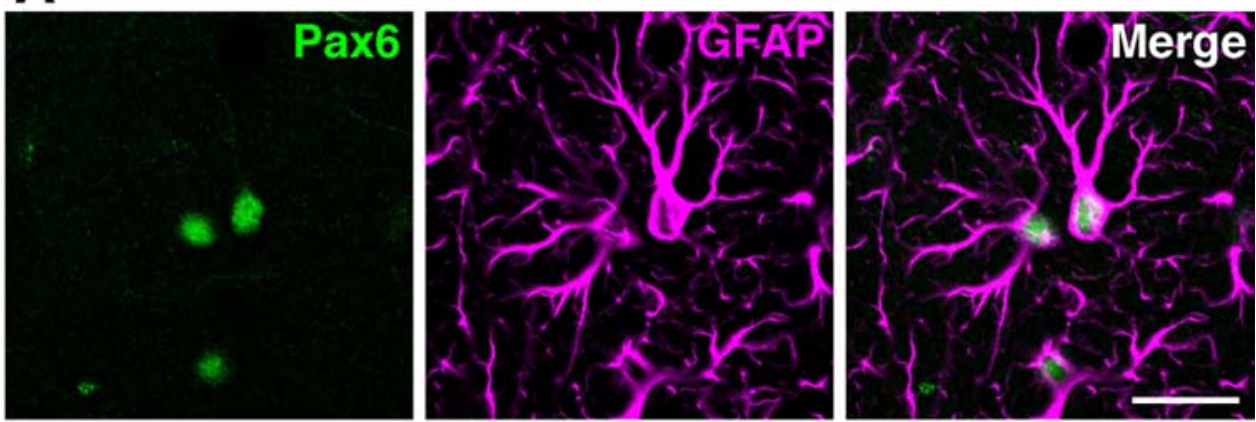

B
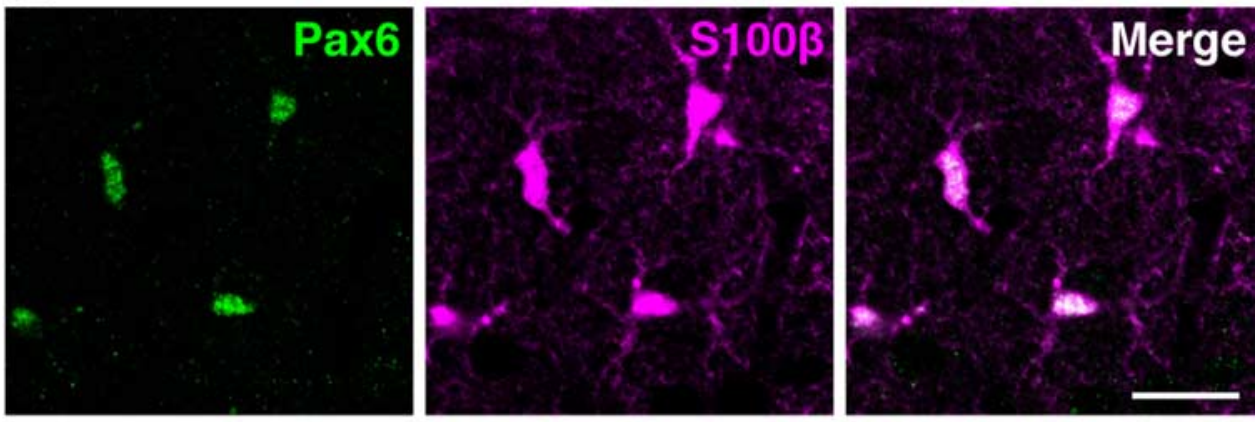

C
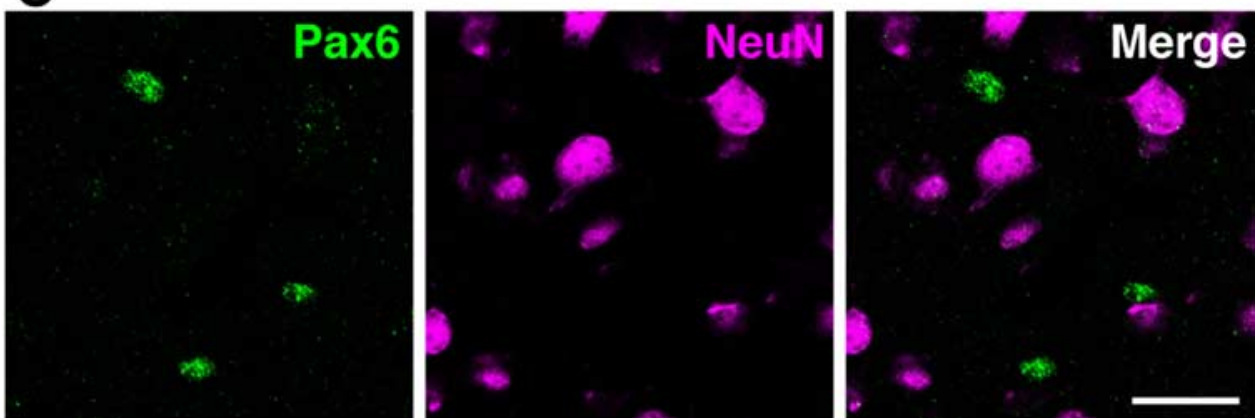

Figure 1. Pax6 is expressed in mature astrocytes in vivo. Double staining of Pax6 (green) together with cell-type-specific markers (GFAP, S100 $\beta$, and NeuN; magenta) in the wild-type (WT) mouse

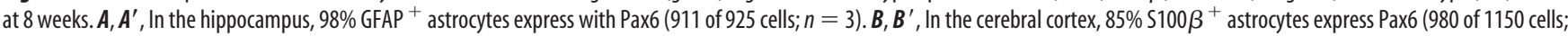
$n=3) . C, C^{\prime}, P^{2} 6^{+}$cells are totally negative for NeuN in the cerebral cortex. Scale bars, $20 \mu \mathrm{m}$.

migration. We also confirmed the results of astrocytes proliferation and maturation in the embryonic spinal cord. Finally, we examined the activity of Akt, a signaling molecule that associates with cell proliferation and migration. Our findings add another pivotal role of Pax6 in the developing CNS.

\section{Materials and Methods}

Mice. Large colonies of heterozygous Pax6 mutant mice (Sey/+) (Hill et al., 1991) and wild-type ICR mice (littermates of Sey/ + mice) were maintained at Tohoku University School of Medicine. To examine the genotypes, PCR analyses for Sey/+ and Sey/Sey were performed as described previously (Swanson et al., 2005). All experimental procedures were approved by Ethics Committee for Animal Experiments of Tohoku University Graduate School of Medicine, and animals were treated according to the National Institutes of Health guidance for the care and use of laboratory animals.

Tissue preparation. The wild-type and Sey/Sey mouse embryos, from embryonic day 14.5 (E14.5) to E18.5, were obtained by laparotomy under terminal anesthesia using pentobarbital sodium. For immunohistochemistry, E14.5 embryos were immersion-fixed with $4 \%$ paraformalde- hyde (PFA) in $0.01 \mathrm{~m}$ PBS (sodium phosphate buffer, $\mathrm{pH} 7.4$ ) for $2 \mathrm{~h}$, and E16.5 and E18.5 embryos were perfused transcardially with 4\% PFA. The brains were cut by a cryostat (Leica, Wetzlar, Germany) into $12 \mu \mathrm{m}$ coronal sections. For immunostaining of Pax6 and astrocytic markers in the adult brain, 8-week-old mice were deeply anesthetized with pentobarbital sodium before being killed, and brains were perfused transcardially with $4 \%$ PFA in $0.01 \mathrm{~m}$ PBS and cut into $100 \mu \mathrm{m}$ coronal sections with a vibratome (Leica).

Immunohistochemistry. Procedures using cryosections were conducted essentially as reported previously (Osumi et al., 1997; Maekawa et al., 2005). Vibratome sections of the brain were washed with PBS three times, incubated for $30 \mathrm{~min}$ in PBS-Triton X-100 containing $2 \%$ goat serum, and stained with anti-Pax6 antibody (Inoue et al., 2000) (supplemental Fig. 1, available at www.jneurosci.org as supplemental material) overnight at $4^{\circ} \mathrm{C}$. The sections were washed with PBS three times, incubated with Alexa Fluor 488 or 555 (Invitrogen, Carlsbad, CA) for $1 \mathrm{~h}$ at room temperature, washed, and mounted on slide glass. Antibodies against GLAST and fatty-acidbinding protein 7 (FABP7) were kind gifts from Dr. Masahiko Watanabe (Hokkaido University, Sapporo, Japan) (Iwanaga et al., 2005) 
and Dr. Yuji Owada (Yamaguchi University, Yamaguchi, Japan) (Owada et al., 2006), respectively.

BrdU labeling analyses. In vivo, timed pregnant mice received a single intraperitoneal injection of 5-bromo-2-deoxyuridine (BrdU) (Sigma-Aldrich, St. Louis, MO) at $50 \mu \mathrm{g} / \mathrm{g}$ body weight $(10 \mathrm{mg} / \mathrm{ml}$ stock, dissolved in $0.9 \%$ saline), and were killed at $1 \mathrm{~h}$ after a single injection. The obtained $12 \mu \mathrm{m}$ frozen sections were incubated in $2 \mathrm{~N} \mathrm{HCl}$ for $11 \mathrm{~min}$ at $37^{\circ} \mathrm{C}$, and washed in TBS-Tween 20 . Incorporated BrdU was detected using the method described previously (Maekawa et al., 2005). For BrdU examination, the numbers of $\mathrm{BrdU}^{+}$cells were measured in five sections per animal. In vitro, astrocytes were plated at $8 \times 10^{4}$ cells/well in 24 -well plates, with 10 $\mu \mathrm{M}$ BrdU treatment for the final $24 \mathrm{~h}$. Four days after plating, cells were fixed with 4\% PFA and labeled with an anti-BrdU antibody (BD Biosciences, San Jose, CA). The number of BrdU ${ }^{+}$cells was measured in five fields per well.

Cell culture and immunostaining. Cell cultures of mouse cortical astrocytes were prepared essentially as described previously (Toyomoto et al., 2004). Cerebral cortices from E18.5 mice were isolated and meninges were removed. The tissue was gently dissociated by trituration with a pipette. Cells were pelleted at $1000 \times g$ for $2 \mathrm{~min}$, resuspended in DMEM supplemented with $10 \%$ FBS, and then plated in a 25 $\mathrm{cm}^{2}$ culture flask. The medium was replaced $24 \mathrm{~h}$ later and changed every $4 \mathrm{~d}$ until confluence. When the cultures reached confluence, astrocytes were removed by trypsinization and replated onto poly-Llysine (PLL)-coated glass coverslips or culture dishes.

For immunostaining, astrocytes derived from the wild-type, Sey/+, and Sey/Sey were plated at $1 \times 10^{5}$ cells/well in a 24 -well plate. Four days after plating, cells were fixed with $4 \%$ PFA and immunostaining was performed. An anti-nestin antibody was purchased from Millipore Bioscience Research Reagents (Temecula, CA). Fluorescent signals were detected using a confocal laser-scanning microscope (Carl Zeiss, Thornwood, NY).

Transfection of plasmid cDNA. Plasmid cDNAs used for transfection were as follows; $p C A X$ and $p C A X$-GFP (kindly provided by the late K. Umesono) (Takahashi and Osumi, 2002), and $p C A X$ Pax6-EnR (kindly provided by M. Kengaku, RIKEN Brain Science Institute, Wako, Japan). Transfections of wild-type astrocytes were performed using an MP100 Microporator (Digital Bio, Seoul, Korea) according to the manufacturer's instructions for optimization and use. As a control, a mixture of $0.1 \mu \mathrm{g}$ of $p C A X-G F P$ and $0.4 \mu \mathrm{g}$ of $p C A X$ was transfected. For functional inhibition of Pax6, a mixture of $0.1 \mu \mathrm{g}$ of $p C A X-G F P$ and $0.4 \mu \mathrm{g}$ of $p C A X-P a x 6-E n R$ was transfected.

Western blot analysis. Western blot analysis was performed essentially as described previously (Ishida et al., 2002), except with a different sample lysis solution. Cultured astrocytes were homogenized in M-PER (Pierce, Rockford, IL) supplemented with a protease inhibitor mixture (Roche, Indianapolis, IN). Antibodies were to Pax6 (Maekawa et al., 2005), GFAP (Sigma-Aldrich), nestin (Abcam, Cambridge, MA), Akt (Cell Signaling, Danvers, MA), phosphorylated Akt (pAkt) (Ser473) (Cell Signaling), and Hsp90 (Transduction Laboratories, Lexington, KY). The antibody
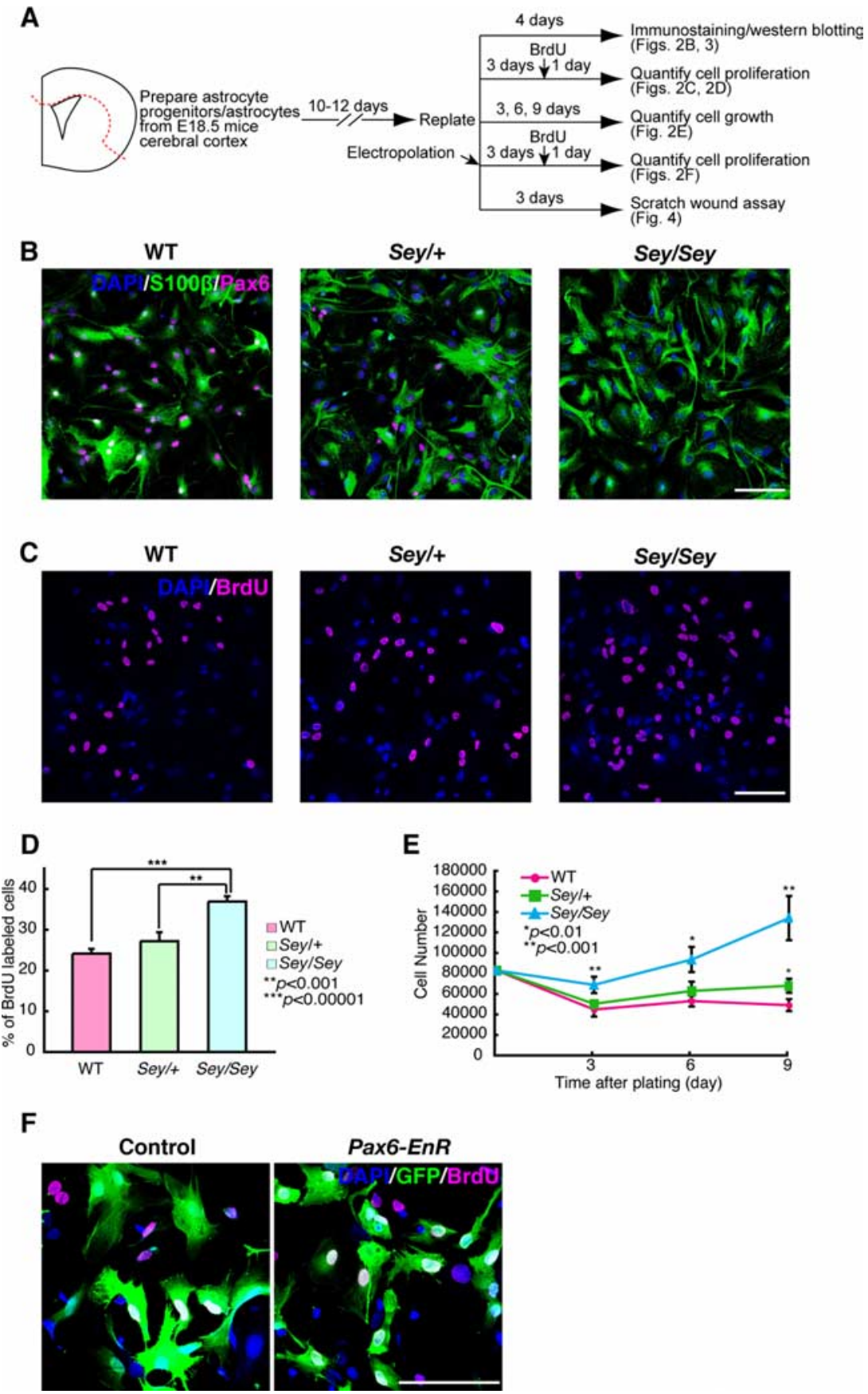

Figure 2. Pax6-deficient astrocytes exhibit aberrant proliferation in vitro. A, Experimental design of cultured astrocytes for different assays shown in Figures 2 - 4. Primary cultures of astrocytes were prepared from WT, Sey/ + , and Sey/Sey mice cerebral cortex at E18.5. After cells became confluent, cells were replated on proper dishes or plates and used for various experiments. $\boldsymbol{B}$, Immunostaining of Pax6 (magenta) and $\mathrm{S} 100 \beta$ (green) in astrocytes derived from the WT, Sey $/+$, and Sey/Sey mice at $4 \mathrm{~d}$ after replating. In astrocytes derived from WT mice, $99 \%$ of $S 100 \beta^{+}$astrocytes are colabeled with Pax6 (1639 of 1643 cells; $n=3$ ). Astrocytes are generated in a Pax6-deficient condition (Sey/Sey). C, D, Quantification of BrdU ${ }^{+}$cells in cultured astrocytes. Astrocytes were cultured for $4 \mathrm{~d}$ with the treatment of BrdU for the final $24 \mathrm{~h}$. The number of BrdU ${ }^{+}$cells is expressed as a percentage of the total number of DAPI-stained cells. The ratio of BrdU ${ }^{+}$(magenta) cells is almostsimilarin astrocytes taken from both WT (24\%) and Sey/ $+(27 \%)$ mice. The number of BrdU ${ }^{+}$cells in Sey/Sey astrocytes (37\%) is quite larger than that in WT ( $p=0.000006$ ) and Sey/ $+(p=0.0006)$ astrocytes. The results are presented as the mean \pm SD of the samples $\left(n=4 ;{ }^{* *} p<0.001\right.$, ${ }^{* * *} p<$ $0.00001)$. E, To evaluate growth rates of WT, Sey/ + , and Sey/Sey astrocytes, the number of DAPI-stained cells is calculated in five fields per well. In WT and Sey/ + astrocytes, the cell number reaches a plateau at $6 \mathrm{~d}$ after plating, and there is no significant difference in the cell numbers between WT and Sey/+ cultures except for those at $9 \mathrm{~d}(p=0.001)$. The cell number of Sey/Sey astrocytes is significantly higher than that of WT and Sey/ + at 3 and $6 \mathrm{~d}$, without reaching a plateau even at $9 \mathrm{~d}(p=0.0009$, 0.001 , and 0.0003 for 3,6 , and $9 d$, respectively). The results are presented as the mean $\pm S D$ of the samples $\left(n=5 ;{ }^{*} p<0.01\right.$, $\left.{ }^{* *} p<0.001\right)$. $F$, To block the function of Pax6 in WT astrocytes, we introduced $p C A X-P a x 6-E n R$ plus $p C A X-$ GFP or $p$ CAX plus $p C A X$-GFP and performed BrdU incorporation assay. Four days after transfection by electroporation, the percentage of BrdU ${ }^{+}$ cells/GFP ${ }^{+}$in pCAX-Pax6-EnR-expressing astrocytes (33.7\%) is larger than that in control astrocytes $(13.7 \%)(p=0.0002)(n=$ 6). Scale bars, $100 \mu \mathrm{m}$. 
A
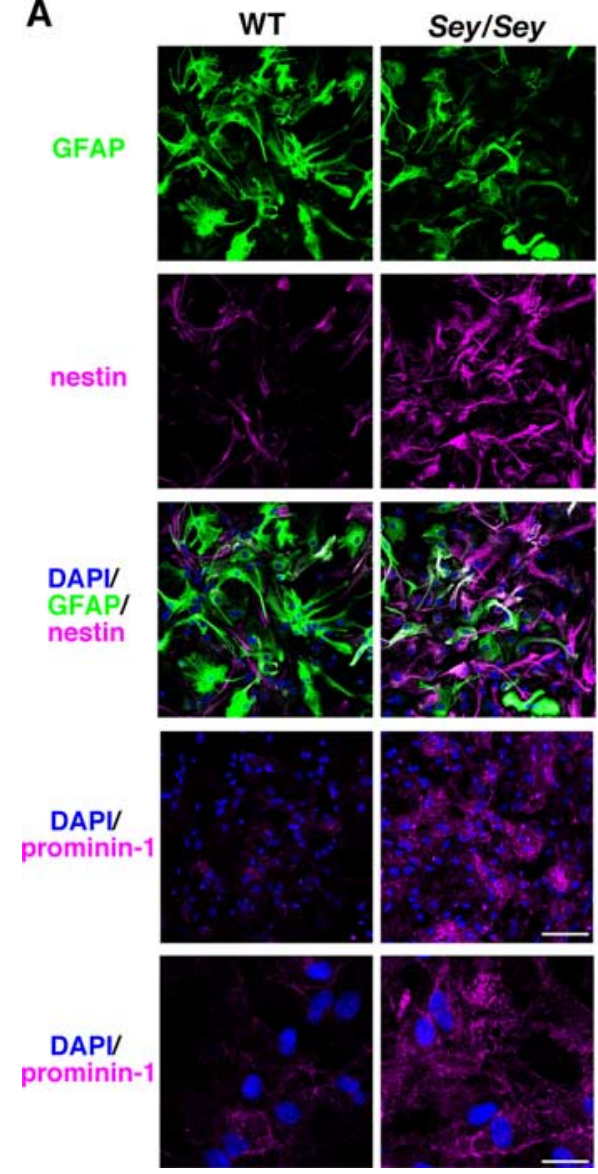

Figure 3. Cultured, Pax6-deficient astrocytes are immature and express neural stem cell markers. $A$, Immunostaining of GAFP, nestin, and prominin-1 (CD133) in WT and Sey/Sey astrocytes. Most WT astrocytes strongly express GFAP, whereas only a small number express nestin or prominin-1. In contrast, a smaller number of Sey/Sey astrocytes express GFAP, and a larger number strongly express nestin and prominin- 1 compared with the WT astrocytes. Scale bar, $100 \mu \mathrm{m}$. The bottom two panels show the high-power images of prominin-1 expression in WT and Sey/Sey astrocytes. Scale bar, $25 \mu \mathrm{m}$. $\boldsymbol{B}$, Cell lysates of cultured WT and Sey/Sey astrocytes were subjected to Western blot analysis with antibodies against GFAP, nestin, prominin-1, and Hsp90. The expression level of GFAP protein is slightly lower in Sey/Sey astrocytes than in WT astrocytes. In contrast, expression levels of nestin and prominin-1, markers for neural stem cells, are higher in Sey/Sey astrocytes than in WT astrocytes. C, Relative expression levels of GFAP, nestin, and prominin-1 protein normalized by Hsp90. The expression level of GFAP protein in Sey/Sey astrocytes is 0.73 -fold lower than that in Sey/Sey astrocytes. The expression level of nestin and prominin-1 protein in Sey/Sey astrocytes is 1.61- and 6.2-fold higher than that in Sey/Sey astrocytes, respectively. The results are presented as the mean \pm SD of samples $(n=3 ; p<0.05)$.

against prominin-1 was a kind gift from Dr. Wieland B. Huttner (Max Planck Institute of Molecular Cell Biology and Genetics, Dresden, Germany) (Weigmann et al., 1997). Immune complexes were detected with the appropriate horseradish peroxidase-conjugated secondary antibodies and ECL Plus (Amersham Biosciences, Piscataway, NJ). Band intensity was measured with an LAS-1000 chemiluminescence imager (Fujifilm, Tokyo, Japan).

Scratch-wound assay. Procedures were conducted essentially as reported previously (Etienne-Manneville, 2006). Cultured astrocytes were seeded in PLL-coated, glass-bottomed dishes (Iwaki, Tokyo, Japan). Medium was replaced $1 \mathrm{~d}$ before scratching. Confluent monolayers of astrocytes were scratched with a sterile plastic pipette tip. Migration of the astrocytes into the scratch was observed under a phase-contrast inverted microscope (Olympus, Tokyo, Japan) and photographed with a CCD camera (Olympus) at 0, 14, 28, 49, and $110 \mathrm{~h}$. The cell-free area was measured using Image J (http://rsb.info.nih.gov/ij/).

Statistical analysis. Two-sided $t$ tests were performed in Microsoft (Redmond, WA) Excel.

\section{Results}

Pax6 is expressed in mature astrocytes in vivo

We have previously shown that the Pax6 transcription factor is expressed by GFAPpositive $\left(\mathrm{GFAP}^{+}\right)$astrocytes in the rat hippocampus (Maekawa et al., 2005). To confirm whether astrocytes generally express Pax6, we first investigated the expression patterns of Pax6 in the postnatal mouse CNS by immunostaining with Pax6 together with the astrocytic markers, GFAP and $\mathrm{S} 100 \beta$. We found that $98 \%$ of $\mathrm{GFAP}^{+}$ astrocytes (911 of 925 cells) in the hippocampus expressed Pax6 (Fig. $1 A, A^{\prime}$ ). In the mouse cerebral cortex, $85 \%$ of astrocytes predominantly expressing $S 100 \beta$ (980 of 1150 cells) coexpressed Pax6 (Fig. $\left.1 B, B^{\prime}\right)$, whereas virtually no $\mathrm{NeuN}^{+}$neurons colabeled with Pax6 (Fig. $1 C, C^{\prime}$ ). Furthermore, astrocytes in other brain regions (e.g., the amygdala and corpus callosum) were also positive for Pax6 (data not shown). In the amygdala and the olfactory bulb, we observed strong Pax6 expression in neurons colabeled with NeuN but negative for astrocytic markers, as previously reported (Kawano et al., 1999; Hack et al., 2005; Kohwi et al., 2005; Tole et al., 2005) (supplemental Fig. 2, available at www. jneurosci.org as supplemental material). Together with our similar observations in the rat (Maekawa et al., 2005) (our unpublished data), we revealed for the first time that astrocytes in the postnatal CNS express Pax6.

\section{Increased cell proliferation in Pax6-deficient astrocytes}

To examine the role of Pax6 in astrocytes, we made primary cultures of astrocytes isolated from the E18.5 cerebral cortex of wild-type, Pax6 heterozygous (Sey/+), and Pax6 homozygous (Sey/Sey) mutant mice (Fig. 2A), because astrocytes are generated around birth in the cerebral cortex and Sey/ Sey mice die soon after birth (Hill et al., 1991). Immunostaining with an anti-Pax6 antibody revealed that Pax6 was expressed in 99\% (1639 of 1643 cells) of cultured astrocytes (marked with $S 100 \beta)$ taken from the wild-type and Sey/ + cortex primordia (Fig. $2 B$ ). Unexpectedly, $S 100 \beta^{+}$astrocytes were generated from Sey/Sey cerebral cortex in vitro (Fig. $2 B$ ), indicating that Pax6 is unnecessary for astrocyte production.

The cultured astrocytes showed a flattened, polygonal shape, and there was slight morphological difference in the cultured astrocytes from all three genotypes (Fig. $2 B$ ). Thus, we assayed the reaction of the cultured astrocytes against the cAMP analog, dibutyryl cAMP (dbcAMP), which stimulates astrocytes to show process-bearing, stellate morphology (Abe and Saito, 1997) (supplemental Fig. $3 A, B$, available at www.jneurosci.org as supplemental material). The percentage of stellate cells after dbcAMP treatment was 21\% (at $6 \mathrm{~h}$ ) and 22\% (at $24 \mathrm{~h}$ ) lower in Sey/Sey 
astrocytes than wild-type astrocytes (supplemental Fig. 3C, available at www. jneurosci.org as supplemental material). This reduced response to dbcAMP in Sey/ Sey astrocytes implies immature differentiation under Pax6-deficient conditions (see below).

We then measured astrocyte proliferation with BrdU incorporation and cell growth assays (Fig. 2A). Four days after plating, the ratio of $\mathrm{BrdU}^{+}$cells was similar in astrocytes from the wild-type (24\%) and Sey/ $+(27 \%)$, but there were dramatically more $(37 \%)$ in Sey/Sey mice (Fig. 2C,D). Cell numbers in the wild-type and Sey/+ cultures reached a plateau $6 \mathrm{~d}$ after plating, with no significant difference in cell numbers until $9 \mathrm{~d}$ in culture (Fig. $2 \mathrm{E}$, magenta circles and green squares). In contrast, Sey/ Sey astrocytes increased in number at 3 and $6 \mathrm{~d}$, without reaching a plateau even at $9 \mathrm{~d}$ (Fig. 2E, blue triangles).

The above results strongly suggest that Pax6 regulates the proliferation of astrocytes. To verify that Pax6 function is truly involved in the molecular mechanism of astrocyte proliferation, we misexpressed a dominant-negative form of $\operatorname{Pax} 6$ [i.e., the DNA-binding domain of Drosophila Engrailed $(E n R)$ is replaced with the transcriptional activation domain of Pax6 (Yamasaki et al., 2001)] in astrocytes derived from wild type by electroporation (Fig. $2 A, F)$. The percentage of $\mathrm{BrdU}^{+} / \mathrm{GFP}^{+}$ cells was significantly higher in Pax6-EnR misexpressed astrocytes (33\%) compared with control $(13 \%)(p=0.0002 ; n=6)$ (Fig. $2 F$ ). This result is quite similar to the astrocytes derived from Sey/Sey (Fig. $2 C, D)$. Despite the role of Pax6 in neurogenesis, in which it maintains proliferation of neural stem/progenitor cells, the elimination of Pax6 function conversely stimulates proliferation in the astrocytes.

\section{Cultured, Pax6-deficient astrocytes are immature and express} neural stem cell markers

The remarkable cell proliferation and reduced reaction against dbcAMP in Pax6-deficient conditions suggested that astrocytes derived from Sey/Sey mice were immature. To clarify this possibility, we investigated the expression of typical markers for mature astrocytes (GFAP) and neural stem cells/astrocytic progenitor cells (nestin) by immunostaining. Most astrocytes derived from the wild type strongly expressed GFAP, whereas only a small population of the wild-type astrocytes (21\%) expressed nestin (Fig. $3 A$ ). In contrast to the wild type, a smaller number of Sey/Sey astrocytes expressed GFAP, and more astrocytes (75\%) strongly expressed nestin (Fig. 3A). Western blotting also confirmed lower GFAP expression (0.73-fold) and higher nestin expression (1.61-fold) in Sey/Sey astrocytes than in the wild-type ones, although these differences in expression were not statistically significant (Fig. $3 B, C$ ). Interestingly, we observed larger population of astrocytes strongly express prominin-1 (CD133), a neural stem

A

Oh
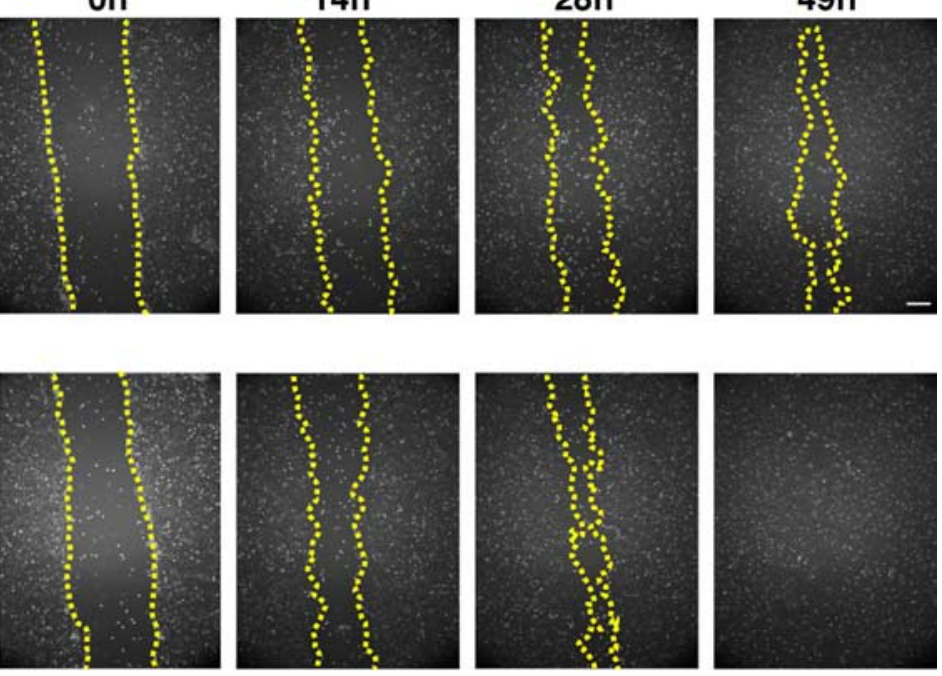

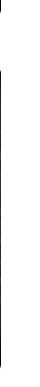

Figure 4. Cultured astrocytes exhibit higher migration potential in the Pax6-deficient condition. Scratch-wound assay was

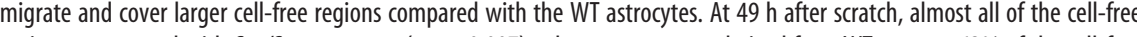
regions are covered with Sey/Sey astrocytes ( $p=0.007$ ), whereas astrocytes derived from WT cover $\sim 62 \%$ of the cell-free regions. The yellow dotted lines represent the edge of the cell-free regions. The results are presented as the mean values \pm SD of the samples $\left(n=3 ;{ }^{*} p<0.05,{ }^{* *} p<0.01\right)$. Scale bar, $500 \mu \mathrm{m}$.

cell marker (Weigmann et al., 1997), in Sey/Sey compared with the wild-type astrocytes (Fig. $3 A$ ). We also confirmed dramatically higher expression (6.2-fold) of prominin-1 in Sey/Sey astrocytes than in the wild-type astrocytes by Western blotting (Fig. $3 B, C)$. The increased expression levels of these neural stem cell markers strongly suggest that Sey/Sey astrocytes are immature and that stem-like cells dominate the population, as in cases of malignant glioma.

\section{Pax6-deficient astrocytes exhibit high migration potential}

Malignant glioma has a particularly high-invasive phenotype, and it virulently invades into surrounding normal brain regions, making complete surgical resection difficult. Because our data thus far suggest glioma-like phenotypes (increased proliferation and immature differentiation) in the astrocytes derived from Sey/Sey, we further hypothesized that migration potential was enhanced in astrocytes with Pax6 deficiency. To test this, we performed a scratch-wound assay in cultured astrocytes (Fig. 2A). At 14 and $28 \mathrm{~h}$ after scratch, Sey/Sey astrocytes robustly migrated and covered larger areas within cell- 

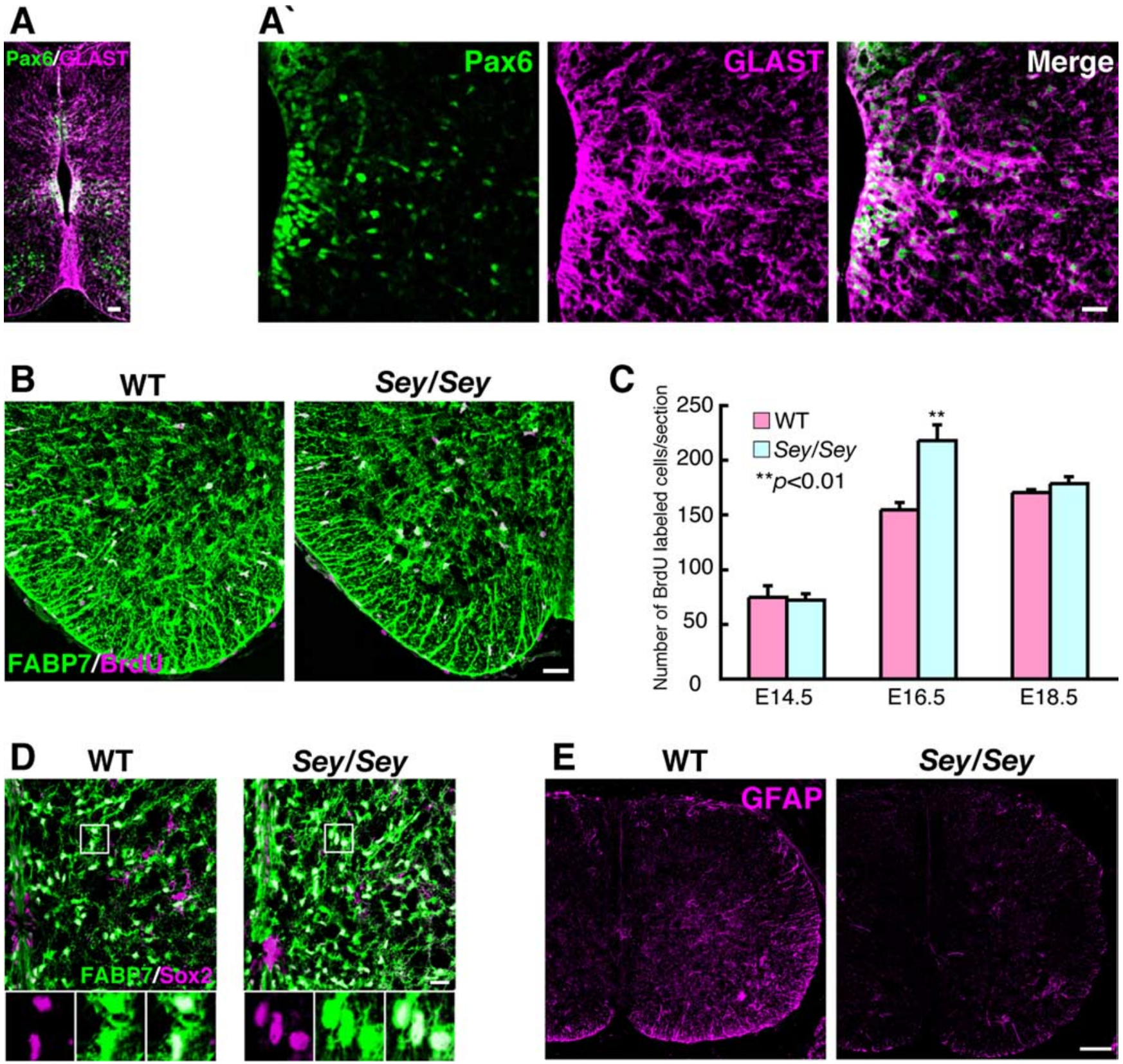

Figure 5. Sey/Sey astrocytes exhibit transiently increased proliferation in the spinal cord. $A, A^{\prime}$, Immunostaining of Pax6 and GLAST in coronal sections of E14.5 mouse spinal cord. Pax6 ${ }^{+}$(green) cells are located in the ventricular zone and gray matter. Almost all Pax6 ${ }^{+}$cells are colabeled with GLAST (magenta). Scale bars, $20 \mu \mathrm{m}$. $\boldsymbol{B}$, Immunostaining of BrdU and FABP7 (BLBP) in coronal sections of 16.5 mouse spinal cord. BrdU ${ }^{+}$cells (magenta) are observed all over the spinal cord, and almost all of these cells coexpress FABP7 (green), a marker for astrocytic progenitors and mature astrocytes, in both WT and Sey/Sey (seen as white cells). Scale bar, $40 \mu \mathrm{m}$. C, Quantification of BrdU ${ }^{+}$cells in WT and Sey/Sey spinal cord at E14.5, E16.5, and E18.5. The number of BrdU ${ }^{+}$cells in the Sey/Sey spinal cord is significantly higher ( $p=0.006$ ) than that in WT at E16.5, whereas there is no statistical difference in the number of BrdU ${ }^{+}$cells between WT and Sey/Sey spinal cords at E14.5 and E18.5. The results are presented as the mean values \pm SD of the samples $\left(n=3 ; *^{* *}<0.01\right)$. D, Immunostaining of Sox2 and FABP7 in coronal sections of E18.5 WT and Sey/Sey spinal cord. The number of Sox2 (magenta) and FABP7 (green) double-positive cells (seen as white cells) are dramatically larger in Sey/Sey spinal cord (1374 cells/section) than that in WT (1149 cells/section) ( $p=0.0003 ; n=3)$. Scale bar, $20 \mu \mathrm{m}$. E, Immunostaining of GFAP in coronal sections of E16.5 WT and Sey/Sey spinal cord. Expression of GFAP in the spinal cord of Sey/Sey mouse is lower than that in WT mouse. Scale bar, $100 \mu \mathrm{m}$.

free regions than the wild-type ones (Fig. $4 A, B$ ). At $49 \mathrm{~h}$ after scratch, $94 \%$ of cell-free regions were covered with Sey/ Sey astrocytes, whereas only $62 \%$ were covered with the wildtype astrocytes (Fig. $4 A, B$ ). Even after $110 \mathrm{~h}$, the wild-type astrocytes did not completely fill in the cell-free regions (data not shown). These results clearly indicate that Sey/Sey astrocytes have grater migration potential than the wild-type astrocytes.
Sey/Sey astrocytes exhibit transiently increased proliferation in the spinal cord

We next tested whether the greater proliferation seen in cultured Sey/Sey astrocytes might also occur in vivo. However, Sey/Sey mice die soon after birth, so the cortex is not a suitable model. We instead investigated proliferation of astrocyte progenitors/astrocytes in the spinal cord, where astrocyte production commences prenatally. First, immunostaining of Pax6 together with GLAST, 
an early astrocytic precursor marker, in the mouse spinal cord at E14.5 showed Pax6 expression in the ventricular zone (containing neural stem cells and neuronal/glial progenitors) and in the gray matter (containing neurons and immature astrocytes at this stage) (Fig. $5 A, A^{\prime}$ ). All Pax6 ${ }^{+}$cells were colabeled with GLAST (Fig. $5 A, A^{\prime}$ ) as observed in our previous report (Ogawa et al., 2005), indicating that astrocyte progenitors express Pax6.

Next, we performed a BrdU incorporation assay in the spinal cord (Fig. $5 B, C$ ). BrdU ${ }^{+}$cells were observed throughout the spinal cord at E14.5, E16.5, and E18.5, with most cells coexpressing FABP7 [brain lipid binding protein (BLBP)], a marker for astrocytic progenitors and mature astrocytes (Barry and McDermott, 2005; Owada et al., 2006) (Fig. 5B). The number of BrdU ${ }^{+}$cells (seen as white cells) in the Sey/Sey spinal cord (217 cells/section) was significantly higher than in the wild type (154 cells/section) at E16.5, but not different at E14.5 or E18.5 (Fig. 5C). We also observed a large number of cells expressing both Sox2, a marker for neural stem cells and astrocytic progenitor cells (Bani-Yaghoub et al., 2006), and FABP7 in the Sey/Sey spinal cord (seen as white cells) compared with those in the wild type at E18.5 (Fig. 5D). Quantitatively, the number of Sox2 and FABP7 double-positive cells in the spinal cord was 1374 cells/section in Sey/Sey, which was significantly larger than 1149 cells/ section in the wild type $(p=0.0003)$ (Fig. 5D). Moreover, we observed lower expression of GFAP in the spinal cord of Sey/Sey mice at E16.5 (Fig. 5E). All of these results indicate a transient increase in proliferation and immature differentiation of astrocytic progenitor cells in Sey/ Sey spinal cord compared with the wild type, further indicating the crucial functions of Pax6 in gliogenesis in vivo.

\section{Pax6-deficient astrocytes have increased Akt activity}

The phenotypes that we observed in Pax6-deficient astrocytes (i.e., aberrant cell proliferation, immature differentiation, increased stem-like cells, and higher migration potential) have a strong resemblance to malignant glioma. Human and mouse gliomas show elevated expression of genes that encode growth factors, their receptors, and downstream signaling molecules, including phosphatidylinositol 3-kinase (PI3K) and Akt (Holland et al., 2000; Sonoda et al., 2001). The PI3K/Akt pathway actively suppresses apoptosis and enhances cellular survival (Bader et al., 2005; Manning and Cantley, 2007). Therefore, we examined protein expression levels of Akt and its activated form, pAkt. Although Akt expression was similar in the wild-type and Sey/Sey astrocytes, expression of pAkt was increased by 1.8 -fold in Sey/Sey astrocytes $(p=0.00003 ; n=$ 5) (Fig. 6A,B). Sey/Sey astrocytes did not show abnormal activation of Erk1/2, a downstream component of the Ras sig-
A

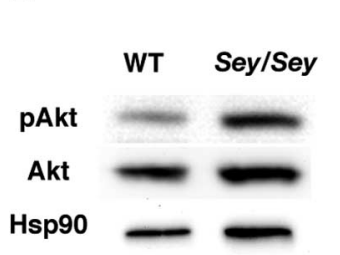

B

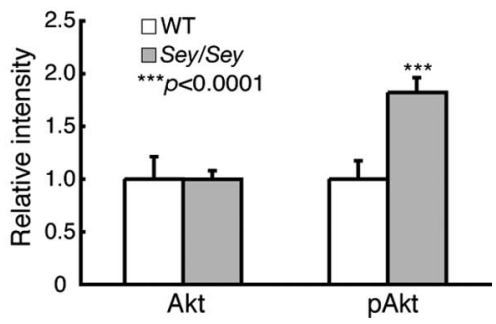

Figure 6. Akt activity is increased in cultured astrocytes in Pax6-deficient condition. $\boldsymbol{A}$, The equal amount of total protein $(10 \mu \mathrm{g})$ from cell lysates of cultured WT and Sey/Sey astrocytes (Fig. $2 A$ ) are subjected to Western blot analysis with antibodies against Akt, pAkt, or Hsp90. The level of expression of pAkt is higher in Sey/Sey astrocytes than in the WT astrocytes. $\boldsymbol{B}$, Relative expression level of Akt and pAkt protein normalized by Hsp90. The expression level of Akt protein in WT astrocytes is similar that in Sey/Sey astrocytes. In contrast, the expression level of pAkt protein in Sey/Sey astrocytes is 1.8-fold higher than that in the WT astrocytes ( $p=$ $0.00003)$. The results are presented as the mean values $\pm S D$ of the samples $\left(n=5 ;{ }^{* * *} p<\right.$ $0.0001)$.
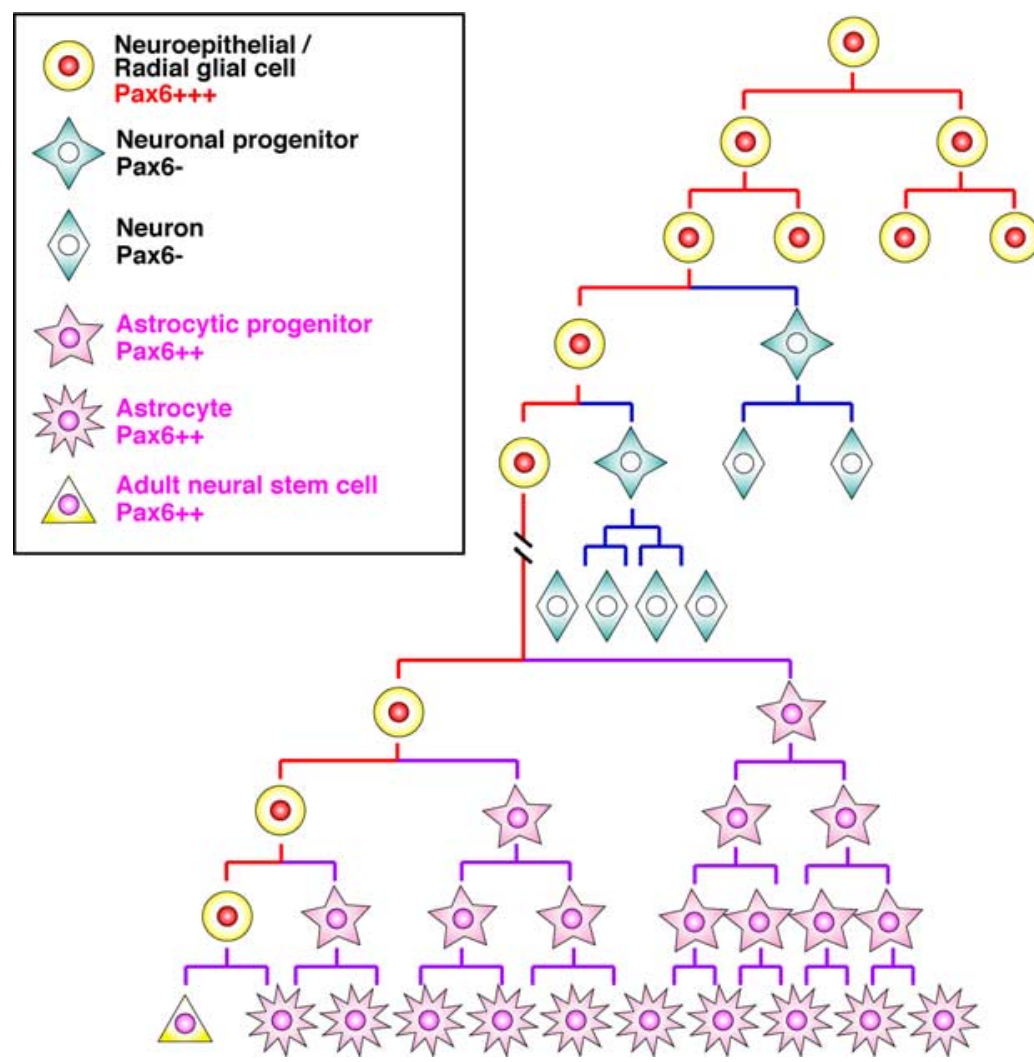

Early

Late

Figure 7. Pax6 expression persists from neuroepithelial/radial glial cells to astrocytes and adult neural stem cell. Schematic illustration of Pax6 expression in the course of cortical neurogenesis and gliogenesis. Neuroepithelial/radial glial cells (Pax6+++ ) initially undergo highly regulated self-renewing divisions and eventually generate lineage-committed progenitor cells, which subsequently differentiate into the three main neural lineages [i.e., neurons (Pax6-), astrocytes (Pax6 ++ ), and oligodendrocytes (data not shown)]. The expression of Pax6 $(++)$ continues in postnatal adult neural stem/progenitor cells. Pax6 expression also persists in astrocytic progenitors (Pax6 ++ ) and in mature astrocytes (Pax6 ++ ). The same expression pattern of Pax6 is also observed in the hippocampus. naling pathway, which is often modified in tumors (data not shown). These data suggest that increased Akt activation mediates, at least in part, increased cell proliferation in Sey/Sey astrocytes. 


\section{Discussion}

Pax6 expression starts in neural stem/progenitor cells in the neural tube from initial CNS development and continues postnatally in those cells in the SGZ (Maekawa et al., 2005; Nacher et al., 2005) and in the SVZ (Hack et al., 2005; Kohwi et al., 2005), the two regions in which adult neurogenesis persists throughout life (Alvarez-Buylla et al., 2002; Gage, 2002). Pax6 expression is also reported in astrocytic progenitor cells within the mouse spinal cord (Ogawa et al., 2005) and in Muller glia in the retina (Bernardos et al., 2007). In the Allan Brain Atlas, Pax6 signal is observed throughout the mouse brain (http://brain-map.org/welcome.do) (Lein et al., 2007), implying the presence of Pax6 expression in astrocytes. Here, we provide firm evidence for the first time that Pax6 is expressed in astrocytes of the entire CNS in vivo and in vitro (Figs. 1, 2), suggesting that Pax6 can be used as a specific marker for astrocytes in the postnatal rodent CNS. Importantly, expression of Pax6 continues from neural stem/progenitor cells in the developing CNS to mature astrocytes and neural stem/ progenitor cells in the adult CNS (Fig. 7). The persistent expression of Pax6 in astrocytes supports the idea that mature astrocytes may retain neural stem cell-like characteristics, as first proposed by Alvarez-Buylla and colleagues (Alvarez-Buylla et al., 2002; Ihrie and Alvarez-Buylla, 2008).

The overlapping expression of Pax6 in embryonic neural stem/progenitor cells, mature astrocytes, and adult neural stem/ progenitor cells led us to ask whether Pax6 has the same function in those types of cells. In embryonic neurogenic regions such as the cortical primordium, the role of Pax6 is complicated. Pax6 can promote neuronal differentiation, implying that Pax6 inhibits cell proliferation (Gotz et al., 1998; Heins et al., 2002). This may be a good explanation for Pax6 function in the amygdala, thalamus, cerebellum, and olfactory bulb, in which Pax6 is expressed by neurons (Kawano et al., 1999; Yamasaki et al., 2001; Hack et al., 2005; Kohwi et al., 2005; Tole et al., 2005). However, loss of Pax6 function reduces the size of neural stem/progenitor pools in the cortical primordium and the hippocampal dentate gyrus (Kawano et al., 1999; Maekawa et al., 2005; Quinn et al., 2007), indicating that Pax6 conversely maintains proliferation of neural stem/progenitor cells. In the present study, we found that loss of Pax6 function either via genetic mutation or transfectional inhibition led to aberrant proliferation and immature differentiation of astrocytes in vitro (Figs. 2, 3) and in vivo (Fig. 5). These phenomena suggest that Pax6 inhibits proliferation of astrocyte progenitors and promotes astrocyte maturation. That is, Pax6 regulates cell proliferation and differentiation in a highly contextdependent manner.

Expression levels of the PAX6 gene are significantly reduced in human malignant glioma samples, suggesting that low levels of PAX6 expression predict an unfavorable prognosis for patients with malignant astrocytic glioma (Zhou et al., 2003). Abnormalities in several receptor tyrosine kinase pathways contribute to the development of gliomas (Holland, 2001; Hulleman and Helin, 2005), including activation of the PI3K/Akt pathway (Chakravarti et al., 2004; Bellacosa et al., 2005). Activated Akt is important in cell proliferation and survival, and abnormal PI3K/ Akt signaling can modulate glioma migration (Joy et al., 2003; Manning and Cantley, 2007). In the present study, we observed higher levels of the activated form of Akt, pAkt (Fig. 6), together with increased proliferation, immature differentiation, and robust migration of cultured astrocytes (Figs. 2-5). Furthermore, we observed fewer dead cells in Sey/Sey astrocytes during the scratch-wound assay experiments (data not shown). These lines of evidence imply that loss of Pax6 function is critical for gliomagenesis.

Pax6 is a transcription factor that exerts its functions through different downstream molecules. We previously reported that Fabp7 is downstream of Pax6 in the developing rat cortex, and is essential for proliferation of neural stem cells during early cortical development (Arai et al., 2005). Furthermore, Pax6 also regulates expression of FABP7 in adult neural stem cells in the rat SGZ (M. Maekawa and N. Osumi, unpublished data). Although FABP7 is expressed in the astrocyte progenitors and mature astrocytes in the wild type (Barry and McDermott, 2005), FABP7 expression does not seem to be decreased in Sey/Sey astrocytes (Fig. 5), suggesting a different regulation of FABP7 gene in astrocytes compared with neural stem/progenitor cells.

In conclusion, our findings that Pax6 works in astrocytes provide solid support for the idea that astrocytes share neural stem cell characteristics. Because Pax6 is pivotal in neurogenesis and gliogenesis, additional studies of downstream targets of Pax6 would provide better understanding of the molecular mechanisms for production of these different cell types in the CNS.

\section{References}

Abe K, Saito H (1997) Developmental changes in cyclic AMP-stimulated stellation of cultured rat cortical astrocytes. Jpn J Pharmacol 75:433-438.

Allen NJ, Barres BA (2005) Signaling between glia and neurons: focus on synaptic plasticity. Curr Opin Neurobiol 15:542-548.

Alvarez-Buylla A, Seri B, Doetsch F (2002) Identification of neural stem cells in the adult vertebrate brain. Brain Res Bull 57:751-758.

Arai Y, Funatsu N, Numayama-Tsuruta K, Nomura T, Nakamura S, Osumi N (2005) Role of Fabp7, a downstream gene of Pax6, in the maintenance of neuroepithelial cells during early embryonic development of the rat cortex. J Neurosci 25:9752-9761.

Bader AG, Kang S, Zhao L, Vogt PK (2005) Oncogenic PI3K deregulates transcription and translation. Nat Rev Cancer 5:921-929.

Bani-Yaghoub M, Tremblay RG, Lei JX, Zhang D, Zurakowski B, Sandhu JK, Smith B, Ribecco-Lutkiewicz M, Kennedy J, Walker PR, Sikorska M (2006) Role of Sox 2 in the development of the mouse neocortex. Dev Biol 295:52-66.

Barry D, McDermott K (2005) Differentiation of radial glia from radial precursor cells and transformation into astrocytes in the developing rat spinal cord. Glia 50:187-197.

Bellacosa A, Kumar CC, Di Cristofano A, Testa JR (2005) Activation of AKT kinases in cancer: implications for therapeutic targeting. Adv Cancer Res 94:29-86.

Bernardos RL, Barthel LK, Meyers JR, Raymond PA (2007) Late-stage neuronal progenitors in the retina are radial Muller glia that function as retinal stem cells. J Neurosci 27:7028-7040.

Blackshaw S, Harpavat S, Trimarchi J, Cai L, Huang H, Kuo WP, Weber G, Lee K, Fraioli RE, Cho SH, Yung R, Asch E, Ohno-Machado L, Wong WH, Cepko CL (2004) Genomic analysis of mouse retinal development. PLoS Biol 2:E247.

Chakravarti A, Zhai G, Suzuki Y, Sarkesh S, Black PM, Muzikansky A, Loeffler JS (2004) The prognostic significance of phosphatidylinositol 3-kinase pathway activation in human gliomas. J Clin Oncol 22:1926-1933.

Cotter DR, Pariante CM, Everall IP (2001) Glial cell abnormalities in major psychiatric disorders: the evidence and implications. Brain Res Bull 55:585-595.

Etienne-Manneville S (2006) In vitro assay of primary astrocyte migration as a tool to study Rho GTPase function in cell polarization. Methods Enzymol 406:565-578.

Gage FH (2002) Neurogenesis in the adult brain. J Neurosci 22:612-613.

Gotz M, Stoykova A, Gruss P (1998) Pax6 controls radial glia differentiation in the cerebral cortex. Neuron 21:1031-1044.

Hack MA, Saghatelyan A, de Chevigny A, Pfeifer A, Ashery-Padan R, Lledo PM, Gotz M (2005) Neuronal fate determinants of adult olfactory bulb neurogenesis. Nat Neurosci 8:865-872.

Heins N, Malatesta P, Cecconi F, Nakafuku M, Tucker KL, Hack MA, Chapouton P, Barde YA, Gotz M (2002) Glial cells generate neurons: the role of the transcription factor Pax6. Nat Neurosci 5:308-315. 
Hill RE, Favor J, Hogan BL, Ton CC, Saunders GF, Hanson IM, Prosser J, Jordan T, Hastie ND, van Heyningen V (1991) Mouse small eye results from mutations in a paired-like homeobox-containing gene. Nature 354:522-525.

Holland EC (2001) Gliomagenesis: genetic alterations and mouse models. Nat Rev Genet 2:120-129.

Holland EC, Celestino J, Dai C, Schaefer L, Sawaya RE, Fuller GN (2000) Combined activation of Ras and Akt in neural progenitors induces glioblastoma formation in mice. Nat Genet 25:55-57.

Hulleman E, Helin K (2005) Molecular mechanisms in gliomagenesis. Adv Cancer Res 94:1-27.

Ihrie RA, Alvarez-Buylla A (2008) Cells in the astroglial lineage are neural stem cells. Cell Tissue Res 331:179-191.

Inoue T, Nakamura S, Osumi N (2000) Fate mapping of the mouse prosencephalic neural plate. Dev Biol 219:373-383.

Ishida N, Hara T, Kamura T, Yoshida M, Nakayama K, Nakayama KI (2002) Phosphorylation of p27Kip1 on serine 10 is required for its binding to CRM1 and nuclear export. J Biol Chem 277:14355-14358.

Iwanaga T, Goto M, Watanabe M (2005) Cellular distribution of glutamate transporters in the gastrointestinal tract of mice: an immunohistochemical and in situ hybridization approach. Biomed Res 26:271-278.

Joy AM, Beaudry CE, Tran NL, Ponce FA, Holz DR, Demuth T, Berens ME (2003) Migrating glioma cells activate the PI3-K pathway and display decreased susceptibility to apoptosis. J Cell Sci 116:4409-4417.

Kawano H, Fukuda T, Kubo K, Horie M, Uyemura K, Takeuchi K, Osumi N, Eto K, Kawamura K (1999) Pax-6 is required for thalamocortical pathway formation in fetal rats. J Comp Neurol 408:147-160.

Kohwi M, Osumi N, Rubenstein JL, Alvarez-Buylla A (2005) Pax6 is required for making specific subpopulations of granule and periglomerular neurons in the olfactory bulb. J Neurosci 25:6997-7003.

Lein ES, Hawrylycz MJ, Ao N, Ayres M, Bensinger A, Bernard A, Boe AF, Boguski MS, Brockway KS, Byrnes EJ, Chen L, Chen L, Chen TM, Chin MC, Chong J, Crook BE, Czaplinska A, Dang CN, Datta S, Dee NR, et al. (2007) Genome-wide atlas of gene expression in the adult mouse brain. Nature 445:168-176.

Maekawa M, Takashima N, Arai Y, Nomura T, Inokuchi K, Yuasa S, Osumi N (2005) Pax6 is required for production and maintenance of progenitor cells in postnatal hippocampal neurogenesis. Genes Cells 10:1001-1014.

Manning BD, Cantley LC (2007) AKT/PKB signaling: navigating downstream. Cell 129:1261-1274.

Nacher J, Varea E, Blasco-Ibanez JM, Castillo-Gomez E, Crespo C, MartinezGuijarro FJ, McEwen BS (2005) Expression of the transcription factor Pax 6 in the adult rat dentate gyrus. J Neurosci Res 81:753-761.

Ogawa Y, Takebayashi H, Takahashi M, Osumi N, Iwasaki Y, Ikenaka K (2005) Gliogenic radial glial cells show heterogeneity in the developing mouse spinal cord. Dev Neurosci 27:364-377.
Osumi N (2001) The role of Pax6 in brain patterning. Tohoku J Exp Med 193:163-174.

Osumi N, Hirota A, Ohuchi H, Nakafuku M, Iimura T, Kuratani S, Fujiwara M, Noji S, Eto K (1997) Pax-6 is involved in the specification of hindbrain motor neuron subtype. Development 124:2961-2972.

Owada Y, Abdelwahab SA, Kitanaka N, Sakagami H, Takano H, Sugitani Y, Sugawara M, Kawashima H, Kiso Y, Mobarakeh JI, Yanai K, Kaneko K, Sasaki H, Kato H, Saino-Saito S, Matsumoto N, Akaike N, Noda T, Kondo H (2006) Altered emotional behavioral responses in mice lacking braintype fatty acid-binding protein gene. Eur J Neurosci 24:175-187.

Prosser J, van Heyningen V (1998) PAX6 mutations reviewed. Hum Mutat 11:93-108.

Quinn JC, Molinek M, Martynoga BS, Zaki PA, Faedo A, Bulfone A, Hevner RF, West JD, Price DJ (2007) Pax6 controls cerebral cortical cell number by regulating exit from the cell cycle and specifies cortical cell identity by a cell autonomous mechanism. Dev Biol 302:50-65.

Simpson TI, Price DJ (2002) Pax6; a pleiotropic player in development. BioEssays 24:1041-1051.

Sonoda Y, Ozawa T, Aldape KD, Deen DF, Berger MS, Pieper RO (2001) Akt pathway activation converts anaplastic astrocytoma to glioblastoma multiforme in a human astrocyte model of glioma. Cancer Res 61:6674-6678.

Swanson DJ, Tong Y, Goldowitz D (2005) Disruption of cerebellar granule cell development in the Pax6 mutant, Sey mouse. Brain Res Dev Brain Res 160:176-193.

Takahashi M, Osumi N (2002) Pax6 regulates specification of ventral neurone subtypes in the hindbrain by establishing progenitor domains. Development 129:1327-1338.

Tole S, Remedios R, Saha B, Stoykova A (2005) Selective requirement of Pax6, but not Emx2, in the specification and development of several nuclei of the amygdaloid complex. J Neurosci 25:2753-2760.

Toyomoto M, Ohta M, Okumura K, Yano H, Matsumoto K, Inoue S, Hayashi K, Ikeda K (2004) Prostaglandins are powerful inducers of NGF and BDNF production in mouse astrocyte cultures. FEBS Lett 562:211-215.

Volterra A, Meldolesi J (2005) Astrocytes, from brain glue to communication elements: the revolution continues. Nat Rev Neurosci 6:626-640.

Weigmann A, Corbeil D, Hellwig A, Huttner WB (1997) Prominin, a novel microvilli-specific polytopic membrane protein of the apical surface of epithelial cells, is targeted to plasmalemmal protrusions of non-epithelial cells. Proc Natl Acad Sci USA 94:12425-12430.

Yamasaki T, Kawaji K, Ono K, Bito H, Hirano T, Osumi N, Kengaku M (2001) Pax6 regulates granule cell polarization during parallel fiber formation in the developing cerebellum. Development 128:3133-3144.

Zhou YH, Tan F, Hess KR, Yung WK (2003) The expression of PAX6, PTEN, vascular endothelial growth factor, and epidermal growth factor receptor in gliomas: relationship to tumor grade and survival. Clin Cancer Res 9:3369-3375. 